\title{
Covering one eye in fixation-disparity measurement causes slight movement of fellow eye*
}

\author{
H.J. SIMONSZ ${ }^{1} \&$ L.J. BOUR ${ }^{2}$ \\ ${ }^{1}$ Abteilung für Schielbehandlung und Neuroophthalmologie, Augenklinik, Kantonsspital, St. \\ Gallen, Switzerland; ${ }^{2}$ The Netherlands Ophthalmic Research Institute, Amsterdam and the \\ Department of Clinical Neurophysiology \& Neurology, Acadernic Medical Centre, \\ Amsterdam, The Netherlands
}

Accepted 15 August 1991

Key words: Eye movements, strabismus, esophoria, exophoria

\begin{abstract}
In the subjective measurement of fixation disparity (FD), the subject fuses contours presented in the peripheral macular areas of both eyes (fusion lock). The position of the eyes relative to each other is monitored by means of two haploscopically seen vertical lines presented in the central macular area, one above and one below a binocularly seen horizontal line. The subject is instructed to shift one of the vertical lines horizontally until the two are aligned, while fixating their intersection with the horizontal line. It has recently been questioned whether the foveolae really are pointed towards the perceived intersection. In this study, we monitored the position of one eye while intermittently covering the fellow eye, while the subject maintained fixation of the intersection of the remaining vertical line and the horizontal line. We found slight differences in position of the measured eye, depending on whether the other eye was covered or not, i.e. depending on the presence or absence of fusion in the macular periphery. These differences were more pronounced in the non-dominant eye.
\end{abstract}

\section{Introduction}

This study addresses the question where exactly the eyes are looking at in the subjective measurement of fixation disparity (FD). For better understanding of the complex mechanisms at work in the FD measurement, a brief review follows.

In the subjective measurement of FD, the subject sees and fuses contours presented in the peripheral macular areas of both eyes ('fusion lock', a square, for instance). The position of the eyes relative to each other is monitored by means of two haploscopically seen objects presented in the central visual field (Fig. 1a). These are usually two vertical nonius lines, one

\footnotetext{
* Presented in part at the Fifth European Conference on Eye Movements, Pavia, September 1989, at the 15th Seminar Week of the Berufsverband der Augenärzte Deutschlands, Arbeitskreis Schielen, Munster, June 1989 and at the meeting of the Schweizerische Ophthalmologische Gesellschaft, Crans Montana, September 1990.
} 


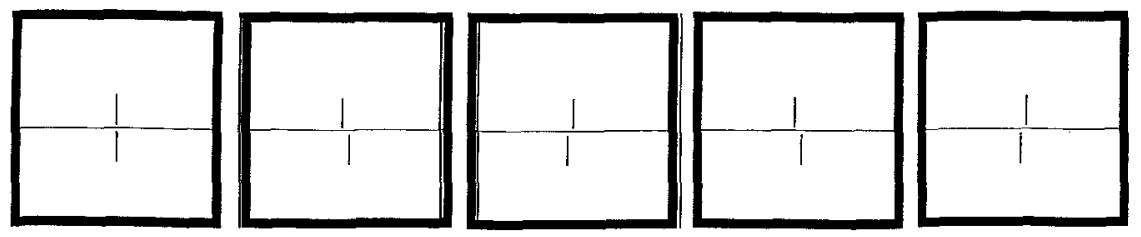

Fig. 1. (a) A schematic view of the pattern seen by the subject during the subjective measurement of FD. The large square is seen binocularly and serves as a fusion lock. In our experiments, the inner size of the fusion lock was $2.5 \times 2.5$ degrees of visual angle. The horizontal line is also seen binocularly, but does not induce fusion. The right eye sees the top vertical nonius line and the left eye the bottom vertical nonius line. The subject is instructed to shift the top nonius line horizontally until the two appear aligned. (b) In reality, the two may well be out of alignment. For instance, when base-in or (c) strong base-out prisms are used. Although most of the fusion is accomplished by vergence, part of it is sensory, i.e. Panum's fusional area is utilized in the macular periphery to overcome a minimal diplopia, represented in this schematic diagram by doubling of the edges of the fusion lock. (d-e) In pathological (obligate or facultative) FD, the nonius lines are set out of alignment by the subject without fusional effort evoked by prisms. Although the fusion lock is fused perfectly, the foveolae are not looking at the same point on the screen. In other words, there is a slight incongruence between the correspondence in the foveolae and the correspondence in the macular periphery.

above the other, separated by a binocularly seen horizontal line. One of the nonius lines can be moved horizontally by the subject, and this is done until the two nonius lines are aligned. For the haploscopically seeing subject the two nonius lines appear aligned, whereas in reality they may well be out of alignment. If they are out of alignment, it means that the foveolae of the eyes are not directed towards the same point on the screen, and FD is said to be present. FD is usually not larger than a few minarc.

FD can be either physiological or pathological. Physiological FD occurs in normal subjects during fusional vergence evoked, for instance, by diverging or by converging prisms. The reason for the occurrence of physiological FD is probably that, during an excessive fusional effort, Panum's area [1] in the peripheral retina is used to fuse the fusion lock, i.e. a small portion of the fusion is not accomplished by a vergence eye movement but by sensory means (Figs. 1b-c). It has been found that this form of FD can change significantly if prisms are worn for longer periods of time. Even after periods as short as 30 seconds, changes do occur $[2,3]$.

Pathological (obligate or facultative) FD occurs spontaneously, without fusional effort evoked by prisms, i.e. although the subject fuses the fusion lock perfectly, the foveolae are not pointed towards the same point on the screen: A slight incongruence exists between the retinal correspondence in the peripheral macular areas and the retinal correspondence in the foveolae (Fig. 1d-e). In other words, perfect fusion of the fusion lock is obtained at a different angle of vergence than perfect fusion of objects in the central visual field. It has been suggested that this pathological FD may be causally 
related to heterophoria and asthenopia [4-10]. However, in a group of 25 visually asymptomatic subjects, only a weak correlation between heterophoria and FD was found [11]. Some optometrists and ophthalmologists prescribe prisms for patients with heterophoria and asthenopia, guided by the FD measurement, and increase the strength of the prisms to the point that the measured FD becomes zero $[12,13]$. At this point, the pathological FD is compensated by the physiological FD. They report that a recurrence of $F D$ is usually observed after the patient has been wearing the prisms for some time and stronger prisms have to be prescribed, sometimes several times, in order to reduce the FD to zero. In our opinion the reason for the recurrence is that, as stated above, physiological FD is highly adaptable, this shedding serious doubt on the validity of this form of treatment. As stated above, the positions of the eyes relative to each other are usually determined by means of two vertical nonius lines, one above and one below a binocularly seen horizontal line. The subject is instructed to align the two vertical lines, while fixating their intersection with the horizontal, binocularly seen, line.

The question which is the subject of this paper is: are the foveolae really pointed at the nonius lines or not? If the localisation of images projected on the retina of one eye does not change with the presence or absence of binocular vision in other parts of the retina, the foveolae will be pointed at the nonius lines at all times, regardless of whether the fellow eye sees or not. Note that this subjective measurement of FD is not disturbed by small saccades, because during saccades both foveolae move equally and the lines therefore stay aligned although they are temporarily not being projected on the foveolae. Hebbard [14] registered the position of both eyes objectively
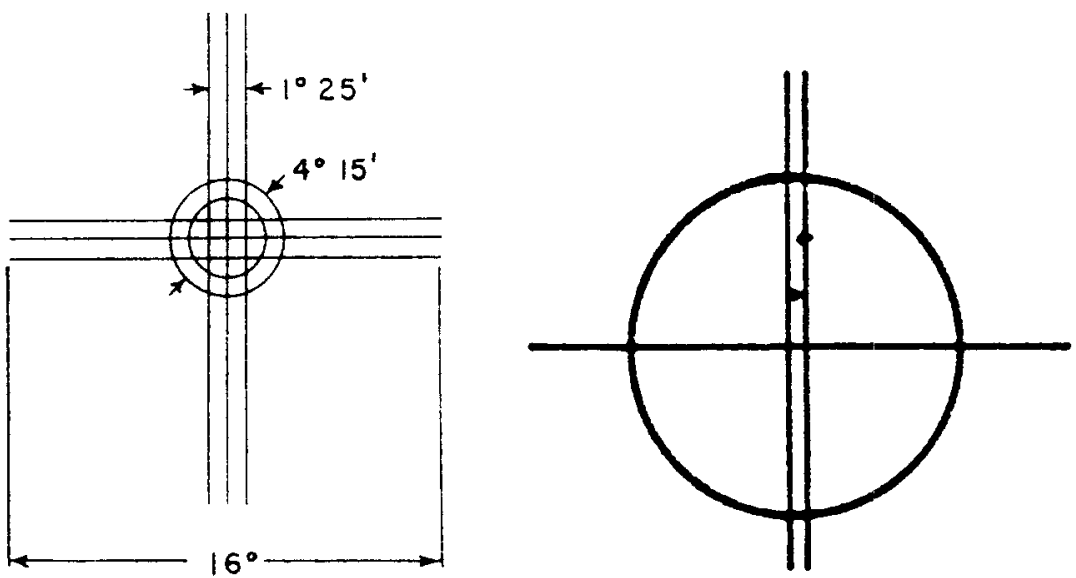

Fig. 2. (a) The fusion locks used by Hebbard and (b) by Bourdy \& Cottin-Lemerle. To monitor eye position, both used 2 projected dots, one for each eye. Hebbard used one dot above and one dot below the centre of the fusion lock, Bourdy \& Cottin-Lemerle used two dots above the centre of the fusion lock. 
during the subjective measurement of FD: One eye was covered intermittently. The subject was instructed to continue fixation of a binocularly seen fixation point. Note that interest was focused, not on how the covered eye moved behind the cover, but on how the position of the non-covered eye varied with the presence or absence of binocular vision (Fig. 3).

It must be noted, however, that Hebbard used an array of horizontal lines, an array of vertical lines and two concentric circles (Fig. 2a) as fusion lock during the subjected measurement of FD, all this being projected in the central visual field. Two dots were seen haploscopically, one above and one below the centre of the fusion lock. They were shifted horizontally by the subject until seen exactly above and below the centre of the fusion lock. The intermittent covering of one eye during the subjective measurement of FD caused, in the other eye, an alternation between the state of monocular fixation of the centre of the fusion lock and the state of binocular fixation of the centre of the fusion lock. Hebbard considered the change in eye position that occurred to be monocular objective FD. He found, in the one subject examined, a close relationship between the sum of the monocular objective FDs of the two eyes and the subjective FD, over a wide range of forced vergence.

Kertesz et al. [15] and Kertesz \& Lee [16] have shed doubt on the reliability of the nonius line estimates. In their experiments, also one eye

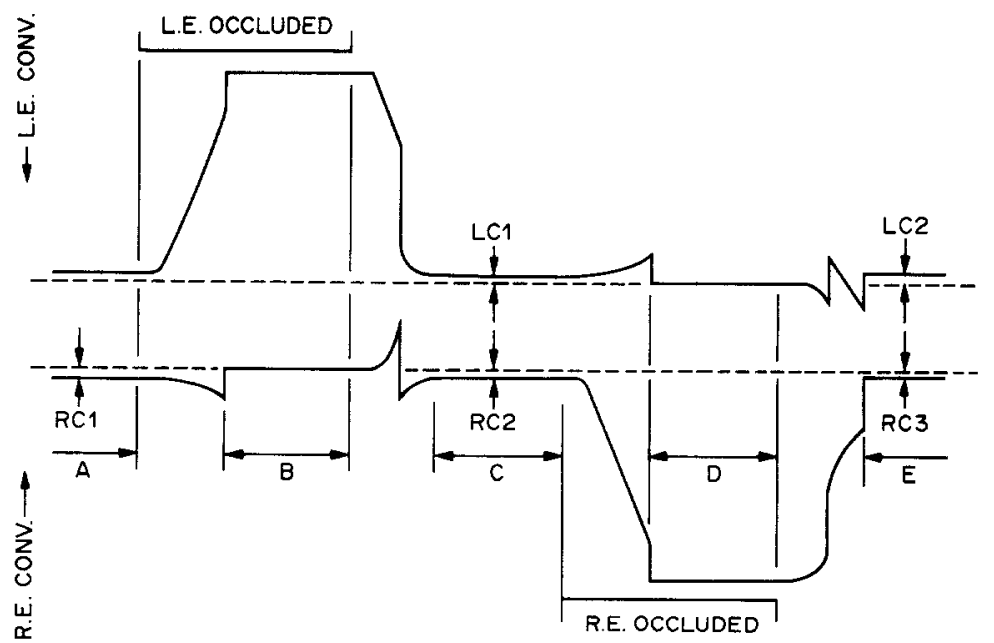

Fig. 3. Diagram by Hebbard to depict the expected eye movements when covering one eye during the subjective FD measurement. The abscissa represents time, the ordinate represents eye movement, the top curve is for the left eye, the bottom curve for the right eye. A base-out prism is in front of one of the eyes and initially (A) fusion is partly accomplished by sensory means ( $\mathrm{RC1}$ ). Then the left eye is covered, and its diverges. Meanwhile (B), the right eye fixates the fixation target perfectly. Then the procedure is repeated covering the right eye. In our experiments, only one eye was covered ten times, and the ten recorded curves were averaged afterwards with the help of a computer. 
was covered intermittently during the subjective measurement of FD (using a peripheral fusion lock), while the position of both eyes was recorded. Considerable differences in eye position were found by Kertesz and Lee depending on whether the fellow eye was covered or not: up to 7.5 minarc in two subjects and up to 42 minarc in a third subject. The mean difference between the position of the non-covered eye under monocular viewing conditions and its position under binocular viewing conditions was called the residual FD ( $\triangle \mathrm{FD}$ ) by Kertesz and Lee. It is important to realize that the $\Delta \mathrm{FD}$ is determined for each eye separately: $\Delta \mathrm{FDl}$ for the left eye during intermittent covering of the right eye and $\Delta \mathrm{FDr}$ for the right eye during intermittent covering of the left eye.

To test statistically the reliability of the subjective FD measurement, Kertesz and Lee determined whether $\Delta$ FD was significantly different from zero in each case (to put it simply, no eye movement occurs when the other eye is covered or, a special case, the movements are compensatory in the two eyes). It must be noted that the subjective FD found in the measurements of Kertesz and Lee was conspicuously large, although the disparity that had to be overcome by fusional vergence was only 2 degrees of convergence. Is it possible that this was caused by the presentation of the fusion lock on an oscilloscope? It is known that FD increases when the spatial frequency of the contours of the fusion lock is decreased [17, 18].

In the measurements by Kertesz \& Lee and in our experiments described below, the intermittent covering caused an alternation between the state of monocular fixation with fusion lock in the macular periphery and the state of monocular fixation without fusion lock. It is essential to be aware of the basic difference that exists between the measurement of monocular 'objective FD' by Hebbard on the one hand and the measurement of $\triangle F D$ by Kertesz \& Lee and our measurements on the other hand. Secondly, a basic difference exists between the measurements of subjective FD by Hebbard and by Bourdy \& Cottin-Lemerle [19] (Fig. 2b, to be discussed) on the one hand and those of Kertesz \& Lee and ourselves on the other hand: In the former the fusion lock is presented centrally, whereas in the latter the fusion lock is in the macular periphery. The former method will not only cause the amplitude of measured FD to be smaller [20] but, in addition, less FD is expected to be found, because the retinal distance between the fusion lock and the haploscopically seen dots is small. In spite of this limitation, Bourdy \& Cottin-Lemerle, employing the same method as Hebbard, did find between 0 and 2 minarc pathological FD in 14 visually asymptomatic subjects, over a wide range of vergence. With Hebbard's and their method, it is possible to determine subjective FD for each eye independently. They found that the monocular components could be in either direction in both eyes, sometimes cancelling each other, and sometimes increasing the amount of total subjective FD. Most interestingly, it was found in 7 out of 14 subjects that the monocular subjective FD was largest in the non- 
dominant eye. In 3 out of 14 , however, it was largest in the dominant eye. We have repeated the measurements of $\triangle F D$ in four subjects by registering the change in mean eye position that occurs when, during the subjective measurement of FD, the fellow eye is covered.

\section{Methods}

Subjects. Four subjects with normal vision participated in the experiments: subject LJB aged 39, subject RAC aged 70, subject WK aged 45 and subject HJS aged 38. The subjects' dominant eyes were HJS: right eye, LJB: right eye (although vision was better in the left eye), WK: left eye, RAC: no eye dominance. RAC had a left esophoria. Full spherical correction was used in subject LJB, the others were emmetropic. In one subject (HJS) measurements were performed with different fusion-lock sizes. The position of the subject's head was firmly secured with a dental bite-board and a headband.

Stimuli. The apparatus employed to measure subjective FD was similar to that used by Ogle [5] (see Fig. 1a for a schematic outline). It was placed $3.42 \mathrm{~m}$ in front of the subject. The fusion lock consisted of a black \& white visual-acuity test card, with a square central black field subtending $2.5 \times 2.5$ degrees of visual angle. In this field no fusion was possible: only a narrow, minarc wide, rear-illuminated, horizontal line was seen binocularly. In one subject (HJS) the experiment was performed for three different field sizes $(1.75 \times 1.75,2.5 \times 2.5$ and $5 \times 5$ degrees $)$, this being achieved by changing the distance between the apparatus and the subject. The two white, rear-projected, vertical nonius lines were 1 minarc wide and 72 minarc long. They were separated from each other by the horizontal, 1 minarc wide line, that was seen binocularly. The nonius lines were presented dichoptically by using crossed polarizers and were rear illuminated by a tungsten light source. The luminance of the nonius lines and the peripheral stimulus was more than $20 \mathrm{~cd} / \mathrm{m}^{2}$. The fusion lock was illuminated by the light in the room. Special care was taken to avoid reflections in the black field surrounding the nonius lines. The upper nonius line could be shifted by the experimenter, following directions given by the subject. To elicit fusional vergence, base-in prisms of different strength were placed in front of the left eye. During the measurement procedure, the nonius lines were first adjusted until the subject saw the two nonius lines in alignment. Then, while the subject maintained fixation of the intersection of the nonius lines and the horizontal line, one of the subject's eyes was intermittently covered by a shutter driven by a servo-motor, while recordings were made of the horizontal positions of both eyes. The duration of the cover-uncover sequence was $8 \mathrm{~s}$. This sequence was repeated 10 times and the recorded eye position 
traces were averaged, at least 6 sequences being averaged. Measurements were performed separately for left and right eyes.

Eye movement recording. Eye position was monitored by the double magnetic induction (DMI) method [21]. This method was extended to record the movements of both eyes. The cross talk between left and right eye position signals for eye positions near the primary position was less than $5 \%$ and could be ignored in these experiments. Ten single recordings of the coveruncover sequence were averaged. The resolution of the system was 2 minarc; the baseline drift was also 2 minarc, resulting in a standard error of mean of 1 minarc in 10 averages. The bandwidth ranged from $0-150 \mathrm{~Hz}$, signals were sampled with a frequency of $170 \mathrm{~Hz}$ and digitally stored in the computer.

Analysis. All computer calculations on the stored recordings were performed in an off-line procedure. Since eye movement registration by the DMI-method is also possible with the eyes closed, eye movements could also be observed during a blink. Blinks could be easily recognized by a large disjunctive movement of both eyes. They were removed from the recordings and replaced by a linear interpolation of the eye position before and after the blink. Micro-saccades were not removed from the recordings. The mean eye position was calculated as the mean over a $3 \mathrm{~s}$ period, starting $1 \mathrm{~s}$ after the covering or uncovering of the fellow eye started (i.e. the last 3 seconds of the 4 second cover or uncover period), in order to exclude artefacts resulting from the initial vergence eye movement after covering or uncovering. $\Delta \mathrm{FDl}$ and $\Delta \mathrm{FDr}$ were computed as the difference between the mean eye position during uncover and the mean eye position during cover of the fellow eye. Subsequently, the mean $\Delta F D l$ and $\Delta F D r$ were calculated as the average of at least 6 single sequences. Finally the total mean $\Delta F D$ was calculated as the sum of $\Delta \mathrm{FDl}$ and $\Delta \mathrm{FDr}$.

\section{Results}

Fig. 4a shows a typical average of 10 cover-uncover sequences during cover of the left eye in subject HJS. A 4-prism-dioptre base-in prism was in front of the left eye. The left panel contains the average eye positions of both eyes averaged from 10 cover periods. The right panel contains the average eye positions of both eyes averaged from 10 uncover periods. Right-eye position during cover of left eye (left upper panel) differs only slightly from right-eye position during uncover of left eye (right upper panel). The left eye makes a convergent eye movement behind the cover (left lower panel), but quickly reverts to the slightly divergent position after uncover (right lower panel). It can be seen that the intruding saccades, performed by both eyes, have been 

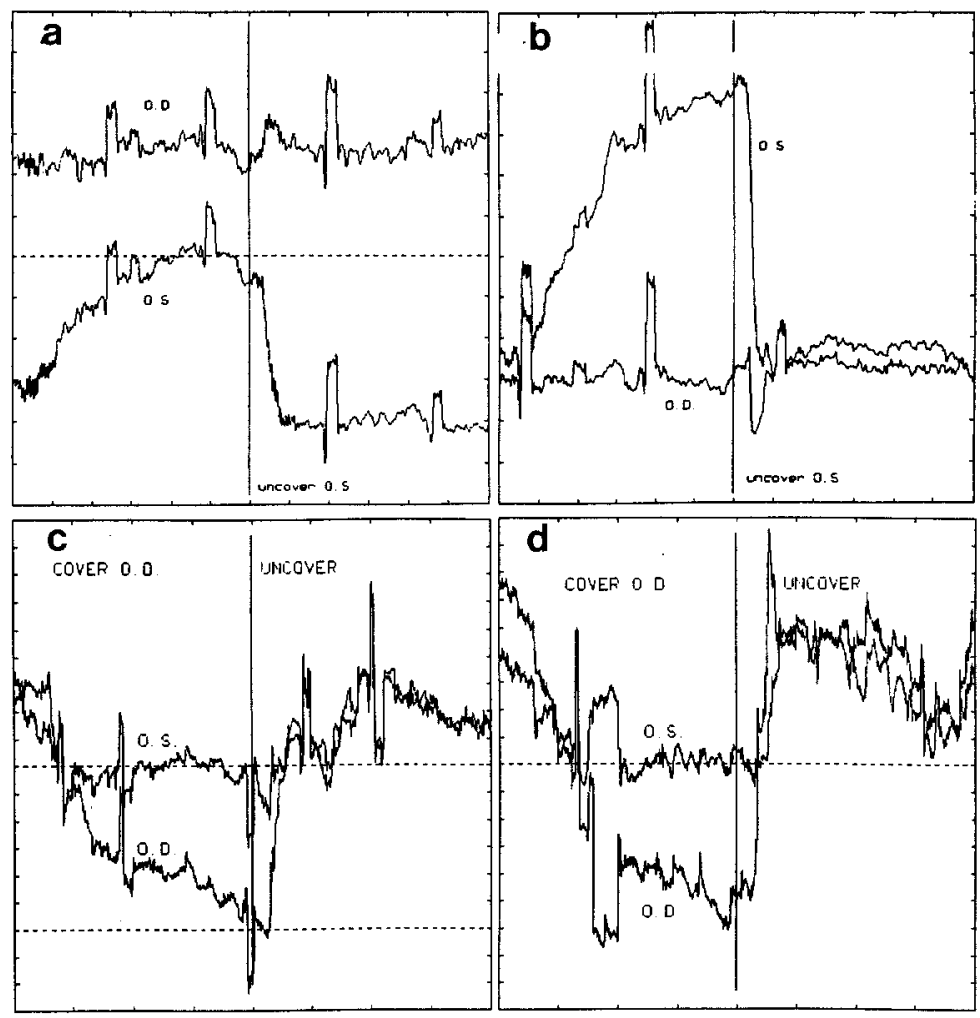

Fig. 4. (a) Typical average of 10 cover-uncover sequences during cover of the left eye in subject HJS. A 4-prism-dioptre base-in prism is in front of the left eye. The entire abscissa represents $8 \mathrm{~s}$. One division of the ordinate represents 5 minarc, an upward deflection of the registration denotes right gaze for both eyes. The left half contains the average eye positions of the right and left eyes averaged from 10 cover periods of $4 \mathrm{~s}$. The right half contains the average eye positions of both eyes averaged from 10 uncover periods of $4 \mathrm{~s}$. Covering of the left eye occurred at the left edge of the figure; uncovering of the left eye occurred at the central vertical line in the figure. Note that right-eye position during cover of left eye (left upper part) differs only slightly from right-eye position during uncover of left eye (right upper part). The left eye makes a convergent eye movement behind the cover (left lower part), but quickly reverts to its slightly divergent position after uncovering (right lower part). It can be seen that the intruding saccades, performed by both eyes, have been reduced by the averaging process. $\Delta F D r$ and $\triangle \mathrm{FDl}$, as presented in the Table, were calculated as the difference between the average eye position (in this case, of the right eye) during the last $3 \mathrm{~s}$ of the $4 \mathrm{~s}$ cover period and the average eye position during the last $3 \mathrm{~s}$ of the $4 \mathrm{~s}$ uncover period. (b) Subject HJS, a 6-prism-dioptre base-in prism is in front of the left eye. The vergence movements of the left eye are larger, but the position of the right eye does not alter much, resembling the results in Fig. 4a. (c) Subject $\mathrm{RAC}$, the right eye is covered intermittently, a 2-prism-dioptre base-in prisms is in front of the right eye. Note that, although the subject is instructed to maintain fixation of the intersection of the remaining vertical nonius and the horizontal line during cover of the right eye, a small left-eye movement does occur when the right eye is covered. (d) Subject RAC, a 4-prismdioptre base-in prism is in front of the right eye. The movement of the left eye that occurs when the right eye is covered, is seen again. 
reduced by the averaging process. The conjugate fast eye movements represent (micro-)saccades. It is evident from this recording that after covering, the covered eye starts to converge to its resting position. After uncovering, this eye returns to the forced divergent position. When a 6-prism-dioptre base-in prism is in front of the left eye (Fig. 4b), the vergence movements of the left eye are larger, but the position of the right eye does not alter much, compared with Fig. 4a. Fig. 4c shows an averaged recording in subject RAC, the right eye being covered intermittently, with a 2-prism-dioptre base-in prism in front of the right eye. Note that, although the subject was instructed to maintain fixation of the intersection of the remaining vertical and the horizontal line during cover of the right eye, a small eye movement did occur when the left eye was covered. When a 4-prism-dioptre base-in prism was put in front of the right eye, the move-

Table 1.

\begin{tabular}{llrlrr}
\hline Subject & Prism & subjective FD & $\Delta$ FDl & $\Delta$ FDr & $\begin{array}{c}\text { Total } \\
\Delta F D\end{array}$ \\
\hline LJB & 0 prism dioptres & $2.5^{\prime} \mathrm{C}$ & $5.0^{\prime} \mathrm{D}$ & $2.0^{\prime} \mathrm{D}$ & $7.0^{\prime} \mathrm{D}$ \\
& 3 & $6.5^{\prime} \mathrm{C}$ & $6.0^{\prime} \mathrm{D}$ & $0.0^{\prime} \mathrm{D}$ & $6.0^{\prime} \mathrm{D}$ \\
& 5 & $230^{\prime} \mathrm{C}$ & $6.0^{\prime} \mathrm{D}$ & $1.0^{\prime} \mathrm{D}$ & $7.0^{\prime} \mathrm{D}$ \\
RAC & 0 & $1.5^{\prime} \mathrm{C}$ & $2.0^{\prime} \mathrm{D}$ & $0.0^{\prime}$ & $2.0^{\prime} \mathrm{D}$ \\
& 2 & $20.0^{\prime} \mathrm{C}$ & $1.0^{\prime} \mathrm{C}$ & $6.0^{\prime} \mathrm{D}$ & $5.0^{\prime} \mathrm{D}$ \\
& 3 & $280^{\prime} \mathrm{C}$ & $2.0^{\prime} \mathrm{C}$ & $12.0^{\prime} \mathrm{D}$ & $10.0^{\prime} \mathrm{D}$ \\
WK & 6 & $5.5^{\prime} \mathrm{C}$ & $1.0^{\prime} \mathrm{C}$ & $2.5^{\prime} \mathrm{C}$ & $3.5^{\prime} \mathrm{C}$ \\
HJS & 0 & $1.0^{\prime} \mathrm{C}$ & $0.0^{\prime}$ & $2.0^{\prime} \mathrm{C}$ & $2.0^{\prime} \mathrm{C}$ \\
& 2 & $3.0^{\prime} \mathrm{C}$ & $3.0^{\prime} \mathrm{D}$ & $1.0^{\prime} \mathrm{C}$ & $2.0^{\prime} \mathrm{D}$ \\
& 4 & $7.0^{\prime} \mathrm{C}$ & $4.0^{\prime} \mathrm{D}$ & $0.0^{\prime}$ & $4.0^{\prime} \mathrm{D}$ \\
\hline
\end{tabular}

Subjective FD, $\triangle F D r, \Delta F D I$ and total $\Delta F D$ for subjects $\mathrm{LJB}, \mathrm{RAC}$, WK and HJS. The distance between the subject and the FD-measurement apparatus was $3.42 \mathrm{~m}$. The square fusion lock (part of a visual acuity test card) surrounded a central black field subtending $2.5 \times 2.5$ degrees of visual angle. In the central black field, two white, vertical, rear-illuminated nonius lines were presented, separated from each other by a narrow, horizontal, rear-illuminated, binocularly seen line. The nonius lines were presented dichoptically by using crossed polarizers. The upper nonius line could be shifted by the experimenter, following directions given by the subject. To elicit fusional vergence, base-in prisms of different strengths were placed in front of the left eye. During the measurement procedure, the nonius lines were first adjusted until the subject saw the two nonius lines in alignment. Then, while the subject maintained fixation of the intersection of the nonius lines and the horizontal line, one of the subject's eyes was covered intermittently while recordings were made of the horizontal positions of both eyes. The duration of the cover-uncover sequence was $8 \mathrm{~s}$. This sequence was repeated 10 times and the recorded eye position was averaged, at least 6 sequences being averaged. Measurements were performed separately for left and right eyes. $\Delta F D I$ and $\Delta F D r$ were computed as the difference between the mean eye position during uncover and the mean eye position during cover of the fellow eye. The total mean $\Delta F D$ was calculated as the sum of $\Delta F D I$ and $\triangle F D r$. The subjects' dominant eyes were HJS: right eye, LJB: right eye, WK: left eye, RAC: no eye dominance. $\mathrm{RAC}$ had a left esophoria. 'C' denotes convergence, ' $\mathrm{D}$ ' divergence. Prism strength is expressed in prism diopters. All other values are expressed in minarc. 
ment of the left eye that occurred when the right eye was covered was still present.

Table 1 shows the subjective FD, the prism used, the mean $\Delta \mathrm{FDl}$, the mean $\triangle F D r$ and the total mean $\triangle F D$ for the 4 subjects at different strengths of base-in prisms. It can be seen from Table 1, that in at least one eye of each subject the $\triangle F D$ does not differ significantly from zero (less than 2 minarcs $=2$ SEM) over a range of divergences. This is within the $95 \%$ confidence limit of the accuracy of the measurement. In the other eyes the $\Delta \mathrm{FD}$ was found to be 2 minarc or more. The mean total $\Delta \mathrm{FD}$ was smaller than 2 minarc in most cases. It was not related to the subjective FD, neither could an association with the direction of vergence, i.e. convergence or divergence, be demonstrated. In two subjects there was a systematic increase in total residual FD with increasing forced divergence, while in one subject the total residual FD remained more or less constant.

In subject HJS, where different fusion-lock sizes were employed, the mean $\Delta$ FDr did not vary for different field sizes (results not shown). With 4 prism-dioptres base-in as diverging stimulus and intermittent cover of the left eye, $\Delta$ FDr remained zero for all field sizes.

\section{Discussion}

Like Kertesz \& Lee, we found slight changes in eye position when the fellow eye was covered during the FD measurement. On the other hand, the magnitude of the change was much smaller than that found in their study. A limitation of our measurements should be emphasized: since we are only able to measure differences in eye position, we do not know exactly what recorded eye position corresponds with the exact projection of the subject's fovea in space. We have to rely on the subject's foveating performance and have no objective information on the position of the fovea.

The most remarkable finding in our measurements was that, in most cases, $\Delta \mathrm{FD}$ was smaller in the dominant eye (dominancy being determined by simple parameters, such as which eye is used for microscopy, etcetera), and not equal to that in the non-dominant eye. Contrary to our findings, Carter [22], in a subjective measurement of FD, found FD to be equally divided between the two eyes in persons with normal binocular vision, and found a smaller FD in the dominant eye only in subjects with a history of partial suppression of one eye. The diagram summarizing his results, however, shows considerable spread. On the other hand, a relationship with dominance has been found by Bourdy \& Cottin-Lemerle, also in a subjective measurement of FD.

What is $\Delta \mathrm{FD}$ ? We think that during binocular vision a localisation map is employed that can be minimally different from the localisation map employed during right-eye vision and minimally different from the localisation 
map employed during left-eye vision. An observation by a patient seen recently with slight bilateral metamorphopsia renders support to this idea: During right-eye vision, a square appeared horizontally elongated to the patient, whereas during left-eye vision it appeared vertically elongated. Surprisingly, during binocular vision the square appeared square to the patient.

It seems possible that slightly anisotropic growth of different parts of the retina, choroid and sclera, after the age that correspondence has been firmly established, may cause slight non-uniformities in the localisation map of one eye, explaining $\triangle F D$. Similarly, slight incongruence of the localisation maps of the two eyes might occur, thus explaining the occurrence of pathological FD. If, for instance, after the age of 10, the foveola of one eye were to shift during growth by only $0.01 \mathrm{~mm}$ relative to the peripheral macular area, pathological FD would inevitably be found.

If different parts of the retina shift during growth in slightly different directions, the magnitude and direction of pathological FD not only varies with the distance between fusion lock and nonius lines [20] but also, to a lesser extent, erratically with the location of the fusion lock. Mastronarde et al. [23] have demonstrated that the retina does grow non-uniformly by comparing the distribution of alpha-type ganglion cells in 3-week old and adult cats. In another study, Milleret et al. [24] found that, on account of the growth of the eye, the distance between the optic disc and the area centralis in kittens decreases from approx. 30 degrees at birth to approx. 15 degrees at 150 days of age. This also partly explains the decrease in size of the receptive fields in early development.

\section{Acknowledgements}

We thank Professor C.M. Schor for his critical review of the manuscript and Professor R.A. Crone for advice and help.

\section{References}

1. Panum PL. In: Untersuchungen über das Sehen mit zwei Augen. Kiel, 1858.

2. Schor CM. The influence of rapid prism adaptation upon fixation disparity. Vis Res 1979; 19: $757-765$.

3. Schor CM. Fixation disparity: a steady state error of disparity-induced vergence. Am J Opt \& Physiol Optics 1980; 57: 618-631.

4. Ogle KN, Mussey F, Prangen, A de H. Fixation disparity and the fusional processes in single binocular vision. Am J Ophthalmol 1949; 32: 1069-1087.

5. Ogle KN. In: Researches in binocular vision. Philadelphia; WB Saunders, 1950.

6. Ogel KN, Prangen A de H. (1951) Further considerations of fixation disparity and the binocular fusional processes. Am J Ophthalmol 1951; 34: 57-72. 
7. Ogle KN, Martens TG, Dyer JA. In: Oculomotor imbalance in binocular vision and fixation disparity. Philadelphia: Lea \& Febiger, 1967.

8. Jampolsky A. Esotropia and convergent fixation disparity. Am J Ophthalmol 1956; 41: $825-833$.

9. Jampolsky A, Flom BC, Freid AN. Fixation disparity in relation to heterophoria. Am J Ophthalmol 1957; 43: 97-106.

10. Crone RA. A new theory about heterophoria. Ophthalmologica 1971; 162: 199-206.

11. Palmer EA, von Noorden GK. The relationship between fixation disparity and heterophoria. Am J Ophthalmol 1978; 86: 172-175.

12. Haase HJ. Fixationsdisparation: Eine erweiterte Theorie und praktische Folgerungen. Der Augenoptiker 1980; 3: 1-9.

13. Pestalozzi D. Über die Behandlung von heterophoren Legasthenikern mit Fixationsdisparation mittels Prismenvollkorrektion. Klin Mbl Augenh 1986; 188: 471-473.

14. Hebbard FW. Comparison of subjective and objective measurements of fixation disparity. J Opt Soc America 1962; 52: 706-712.

15. Kertesz AE, Hampton DR, Sabrin HW. The unreliability of nonius line estimates of vertical fusional vergence performance (letter). Vis Res 1983; 23: 295-297.

16. Kertesz AE, Lee HJ. The nature of sensory compensation during fusional response. Vis Res 1988; 28: 313-322.

17. Schor CM, Wesson M, Robertson KM. Combined effects of spatial frequency and retinal eccentricity upon fixation disparity. Am J Opt \& Physiol Optics 1986; 63: 619-626.

18. Irving EL, Robertson KM. Monocular components of fixation disparity: the effects of monocular image degradation. Invest Ophthalmol 1990; 31 (ARVO Supplement): 95.

19. Bourdy C, Cottin-Lemerle F. La disparité de fixation: variations des composantes monoculaires en fonction de la distance d'observation. Annales Oculist (Paris) 1971; 204: $697-730$.

20. de Decker W, Scheffel T. Simultaneous investigation of fixation disparity, stereo threshold and horopter balance. Graefes Arch klin experim Ophthalmol 1978; 207: 97-109.

21. Bour LJ, van Gisbergen JAM, Bruijns J, Ottes FP. The double magnetic induction method for measuring eye movements - results in monkey and man. IEEE Transactions on Biomedical Engineering 1984; BME-31, 419-427.

22. Carter DB. Studies in fixation disparity. Am J Opt Arch Am \& Acad Optometry 1960; 37 : 408-419.

23. Mastronarde DN, Thibeault MA, Dubin MW. Non-uniform postnatal growth of the cat retina. J Comp Neurol 1984; 228: 598-608.

24. Milleret C, Buisseret $P$, Gary-Bobo E. Area centralis position relative to the optic disc projection in kittens as a function of age. Invest Ophthalmol 1988; 29: 1299-1305.

Address for correspondence: H.J. Simonsz MD, Abteilung für Schielbehandlung, Augenklinik, Kantonsspital, CH-9007 Sankt-Gallen, Switzerland. Tel 41.71.262821. 